\title{
Continuous Preparation of Polymer Coated Drug Crystals by Solid Hollow Fiber Membrane-Based Cooling Crystallization
}

\author{
Dengyue Chen ${ }^{\mathrm{a}}$, \\ Dhananjay Singh ${ }^{\mathrm{a}}$, \\ Kamalesh K. Sirkar ${ }^{a} *$ \\ ${ }^{a}$ Otto York Department of Chemical, Biological and Pharmaceutical Engineering \\ New Jersey Institute of Technology \\ University Heights \\ Newark, NJ 07102 USA \\ Robert Pfeffer ${ }^{\mathrm{b}}$ \\ ${ }^{\mathrm{b}}$ School for Engineering of Matter, Transport and Energy \\ Arizona State University \\ Tempe, AZ 85287 USA
}

\footnotetext{
*To whom correspondence should be addressed: 973-596-8447(tel.); 973-6424854(fax).

Email addresses: dc224@njit.edu (Dengyue Chen); ds64@njit.edu (Dhananjay Singh); sirkar@njit.edu (Kamalesh K. Sirkar); Robert.Pfeffer@asu.edu (Robert Pfeffer)
} 
Abstract: A facile way to continuously coat drug crystals with a polymer is needed in controlled drug release. Conventional polymer coating methods have disadvantages: high energy consumption, low productivity, batch processing. A novel method for continuous polymer coating of drug crystals based on solid hollow fiber cooling crystallization (SHFCC) is introduced here. The drug acting as the host particle and the polymer for coating are Griseofulvin (GF) and Eudragit RL100, respectively. The polymer's cloud point temperature in its acetone solution was determined by UV spectrophotometry. An acetone solution of the polymer containing the drug in solution as well as undissolved drug crystals in suspension were pumped through the tube side of the SHFCC device; a cold liquid was circulated in the shell side to rapidly cool down the feed solution-suspension in the hollow-fiber lumen. The polymer precipitated from the solution and coated the suspended crystals due to rapid temperature reduction and heterogeneous nucleation; crystals formed from the solution were also coated by the polymer. Characterizations by scanning electron microscopy, thermogravimetric analysis, laser diffraction spectroscopy, X-ray Diffraction, Raman spectroscopy, and dissolution tests show that a uniformly coated, free-flowing drug/product can be obtained under appropriate operating conditions without losing the drug's pharmaceutical properties and controlled release characteristics.

Keywords: Polymer coating; Drug crystals; Membrane-based cooling crystallization; Solid hollow fiber 


\section{Introduction}

Polymers are of great importance in controlled release of drugs (Bernkop-Schnurch et al., 2000; Bhattacharyya et al., 2012; Cui et al., 2014; Fluri et al., 2008; Ghebaur et al., 2012; Kakizawa et al., 2010; Laga et al., 2012; Li et al., 2014; Mei et al., 2012; Nahrup et al., 2004; Paulsson and Edsman, 2002; Ramyadevi and Rajan, 2015; Sundaresan et al., 2014; Wulff and Leopold, 2014). A few of the common techniques employed to achieve controlled release of drugs are as follows (Langer, 1990). Diffusion of a drug takes place from a drug reservoir through a polymer layer surrounding the reservoir. Alternately, the drug is dispersed in a polymeric matrix through which it diffuses. The matrix can also ultimately swell or is degraded leading to controlled release of the drug. In the first technique, drugs such as proteins and peptides are protected from degradation by stomach acids by the polymeric coating. Coated with a layer of polyethylene glycol, drug nanoparticles have been found to penetrate a compromised blood-brain barrier (Nance et al., 2012) or overcome a mucous barrier (Lai et al., 2008). Here we are therefore concerned with a continuous fabrication technique involving development of a polymeric coating around the drug particle/crystal.

Methods for developing a polymeric coating around micron-size, submicron particles and nanoparticles may be classified (Wang et al., 2004) as dry or wet, batch or continuous. Techniques studied extensively over the last 20 years e.g., Rapid Expansion of Supercritical Solutions (RESS) (Tsutsumi et al. (1995); Kim et al. (1996)), compressed anti-solvent (Falk et al., 1997), Supercritical Anti-Solvent (SAS) (Wang et 
al., 2004), and Gas Anti-Solvent (GAS) processes, are very demanding due to the very high pressure often involving supercritical $\mathrm{CO}_{2}$ and the low solubility of the polymers in such fluids. Further, these are batch processes as in all industrial crystallization techniques whether they employ cooling, evaporation or anti-solvent to bring about crystallization from a solution.

Conventional crystallization devices containing impeller mixing-based equipment (Myerson, 2002; Tavare, 1989) invariably suffer from poor mixing resulting in extreme variations in the crystallized products. In processes utilizing anti-solvent crystallization, the impinging-jet mixer (Midler et al., 1994) employing two jet streams meeting head to head generates nuclei/crystals which undergo growth subsequently in a well-mixed vessel or a tubular precipitator The shortcomings of this technique have been summarized (Zarkadas and Sirkar, 2006).

To overcome most of the deficiencies of previous techniques, we have adapted the solid hollow fiber cooling crystallization (SHFCC) method developed earlier (Zarkadas and Sirkar, 2004). In the SHFCC, a solution of the drug to be crystallized flows in the bore of a solid hollow fine fiber whose wall is impervious; a cold liquid flows on the shell side of this hollow fine fiber in a countercurrent direction setting up a rapid and highly efficient heat exchange (Song et al., 2010) leading to cooling-based crystallization of the solute in the lumen-side liquid.

Such a technique was employed recently by us (Chen et al., 2014a) to generate a thin coating of the polymer Eudragit RL 100 or poly (D,L-lactide-co-glycolide) (PLGA) 
from its solution in acetone on $550 \mathrm{~nm}$ submicron silica particles; the silica particles were in suspension which was flowing continuously through the bore of the hollow fine fibers. The polymer coating thickness could be varied by changing the operating conditions. By using two different hollow fiber modules having different numbers of hollow fibers, we demonstrated facile scale-up yielding essentially the same polymer coating thickness on the silica particles at two different production rates. Continuous and reproducible polymer coating of silica nanoparticles of $12 \mathrm{~nm}$ diameter in suspension in an acetone solution of the polymer Eudragit RL 100 (Chen et al., 2014b) was also demonstrated. The polymer-coated submicrometer or nano-sized particles were recovered to produce free-flowing particles.

These silica particles were acting as surrogates for drug particles. There are a few other factors to be considered when drug crystals are to be coated continuously by a polymer by the SHFCC technique. For example, the drug may be in solution or it may be present as crystals/particles in suspension or the drug may be present simultaneously in solution as well as in a suspension depending on its solubility. Whenever the drug is present in a solution, it is often a challenge to prevent co-precipitation of the drug and the polymer. For example in the SAS process, there are quite a few examples where co-precipitation took place (co-precipitation of ethyl cellulose and ampicillin (Montes et al., 2012); coprecipitation of polyvinylpyrolidone and diflunisal (Zahran et al., 2014); poly- $\varepsilon$ caprolactone co-precipitated with green tea polyphenols (Sosa et al., 2011). 
The SHFCC technique will always have the polymer in solution in a given solvent. If the drug crystals are introduced into the polymer solution, there is a possibility that the drug may have limited/low solubility in the solvent. On the other hand, if we have a solution of the drug, the polymer may be introduced into the solution and completely dissolved into it. Therefore the most general situation will have both the drug and the polymer in solution; but if the drug has limited solubility, some drug may be present as a suspension. Here we have a solution of the coating polymer which contains in addition a solution of the drug to be crystallized as well as a suspension of micron-sized drug crystals flowing in the solid hollow fine fiber bore. The drug under consideration is Griseofulvin; the polymer used to coat the drug crystals is Eudragit RL 100. The goal of this work is to introduce a facile cooling-based method for continuous polymer coating of drug crystals/particles when the drug is present in a solution as well as in a suspension and the polymer is in solution.

It is useful to identify another hollow fiber membrane-based method for continuous crystallization, the porous hollow fiber-based anti-solvent crystallization (PHFAC) (Zarkadas and Sirkar, 2006). The PHFAC technique has also been employed to develop continuously a suspension of polymer-coated drug particles/crystals from a solution of the drug and the polymer in the solvent by exposure to an anti-solvent (Chen et al., 2015). In this technique, the acetone solution of the drug and the polymer flows on the shell side of a porous hydrophilic hollow fiber. Water acting as the anti-solvent is introduced from the tube side of the hollow fiber through the pores in the hollow fiber 
wall into the hollow fiber shell side where the acetone solution of the polymer and the drug is flowing. Very high supersaturation is created instantaneously by this process leading to rapid crystallization of the drug first followed by nucleation/precipitation of the polymer around the drug crystals.

However, in the PHFAC technique of Chen et al. (2015), both the drug and the polymer were in solution whereas here the drug is present in solution as well as in suspension. It would also be of interest to determine how the results of the PHFAC process compare with what we propose here employing simple cooling without the complexities of an anti-solvent system.

\subsection{Solid Hollow Fiber Cooling Crystallizer}

A single polymeric solid hollow fiber is shown in Figure 1(a). This solid hollow fiber serves as a typical heat exchange tube except the dimensions of the solid hollow fine fiber are orders of magnitude smaller than those of a conventional heat exchange tube. The feed liquid flowing into the hollow fiber lumen is an acetone solution of the drug and the polymer at say, $45^{\circ} \mathrm{C}$. This solution contains a suspension of GF microcrystals as well. As this solution-suspension is pumped through the hollow fiber lumen, it is rapidly cooled down by the cold liquid circulating on the shell side of the hollow fiber module after introduction at $-9.1^{\circ} \mathrm{C}$. The temperature of the liquid exiting the hollow fiber lumen is around $5^{\circ} \mathrm{C}$. The cooling leads to crystallization of the dissolved drug as well as polymer precipitation around the preexisting drug crystals in suspension and the 
rapidly forming drug crystals which allow heterogeneous nucleation of the polymer around them.

The hollow fine fiber was made of polypropylene (PP); it has considerable $\mathrm{pH}$, chemical and solvent resistance as well as mechanical strength. The nonporous fiber wall has a relatively smooth surface which minimizes the possibility of clogging problems inside of the fiber. Figure 1(b) illustrates a solid hollow fiber cooling crystallization (SHFCC) module that has a large numbers of hollow fibers. The number of hollow fibers can be easily adjusted according to the needs of scale up; as the number of hollow fibers increases the diameter of the shell is also increased.

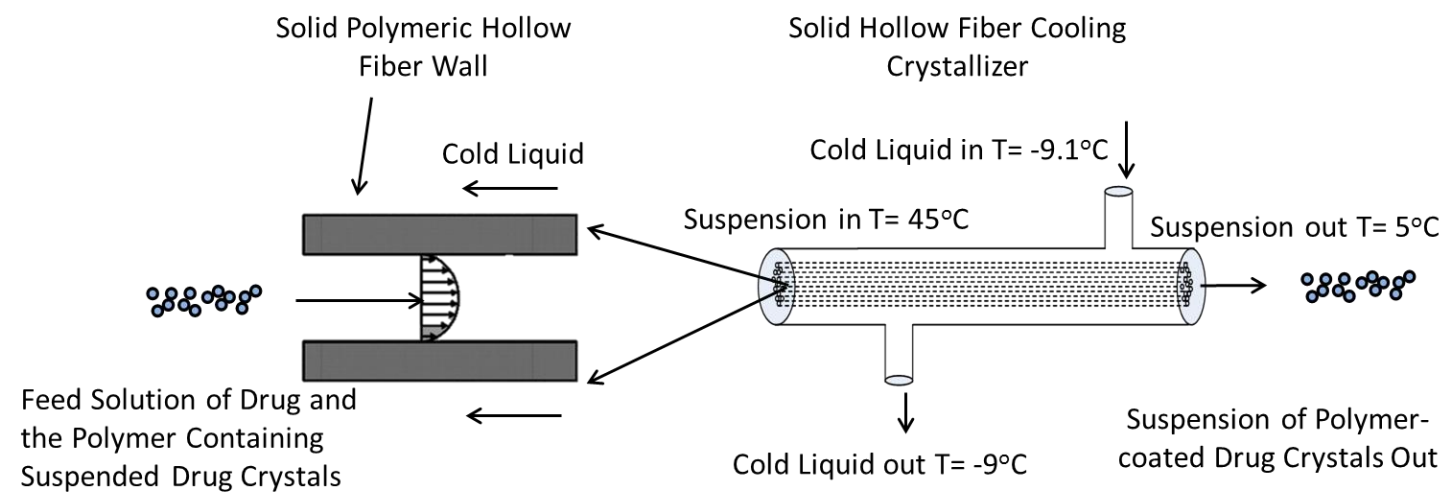

(a)

(b)

Fig. 1. (a) Single solid hollow fiber heat exchanger; (b) Solid hollow fiber membrane based cooling crystallizer module.

\section{Materials and Methods}

\subsection{Materials}

Eudragit RL $100\left(\mathrm{M}_{\mathrm{w}}, 150,000\right.$; a copolymer of methyl methacrylate, ethyl acrylate and methacrylic acid ester) was purchased from Evonik-Degussa (Parsippany, NJ, USA). Drug particles of Griseofulvin (mean particle size $\sim 10 \mu \mathrm{m}$ ) obtained from Letco 
(Decatur, AL, USA) were used as host crystals without further treatment. Its solubility in acetone at $25^{\circ} \mathrm{C}$ is $0.116 \mathrm{M}$. In our experiments, we added an amount of GF that exceeded the solubility limit so that the GF was only partially dissolved. The surfactant sodium dodecyl sulfate (SDS) was from Sigma-Aldrich (St. Louis, MO, USA).

\subsection{SHFCC Module Fabrication}

Solid hollow fine fibers made of PP with the ID/OD of 420/575 $\mu \mathrm{m}$ (Celgard, Charlotte, NC, USA) were used to fabricate the SHFCC module. A total of 46 hollow fine fibers each having an effective length of $47 \mathrm{~cm}$ was placed inside a fluorinated ethylene propylene (FEP) tubing acting as the shell of the module; the hollow fibers act as the tube side of the shell-and-tube type module. The internal diameter of the FEP tubing was $\sim 14 \mathrm{~mm}$. Details describing of the construction of the module can be found in Chen et al (2014a).

A few words here about the hollow fiber dimensions are useful. Generally available polymeric hollow fibers have ID dimension varying between $\sim 250 \mu \mathrm{m}$ and $900 \mu \mathrm{m}$. There are two reasons for selecting the ID dimension used here. First, the ID must be at least 1 to 1.5 orders of magnitude larger than the largest particle dimension in suspension to prevent clogging. Griseofulvin crystal dimensions obtained were around $10 \mu \mathrm{m}$. Therefore the hollow fiber ID selected is more than an order of magnitude larger.

On the other hand, the smaller the ID the better is the temperature control for cooling crystallization. The smaller the ID the lower is the difference in the temperature between the wall and the centerline leading to a much reduced level of difference in the 
supersaturation levels at different radial locations (Zarkadas and Sirkar, 2004). Therefore the dimension of the ID selected is a compromise between two conflicting requirements. Further, the wall thickness should not be large to ensure rapid heat transfer; here a wall thickness of $\sim 75 \mu \mathrm{m}$ provides rapid heat transfer without reducing the fiber strength too much.

\subsection{Experimental Methods}

To determine the range of temperature reduction needed for the SHFCC process, cloud point measurements were first carried out for the polymer Eudragit RL 100 in an acetone solution (containing a small amount of water) before undertaking any coating experiment using the hollow fiber module. The polymer cloud point was determined by a transmissivity test using a UV spectrophotometer (Cary 50; Agilent, Santa Clara, CA) having a temperature control module in the visible wavelength range of 550-800 $\mathrm{nm}$. Details of the relation between cloud point temperature and the ratio of acetone/water are provided in Chen et al. (2014a). A cloud point temperature of $15^{\circ} \mathrm{C}$ was selected; it is easily achieved in the laboratory for cooling crystallization experiments; correspondingly, the ratio of acetone/water in $\mathrm{ml} / \mathrm{ml}$ for this cloud point was $2.5 / 0.5$.

A schematic diagram of the set-up for cooling crystallization-based continuous polymer coating of preexisting drug crystals in suspension as well as synthesis of polymercoated drug crystals is shown in Figure 2. An amount of $2.4 \mathrm{~g}$ of Eudragit RL100 granules was first placed into a flask containing $20 \mathrm{ml}$ acetone solution and kept under stirring for 30 min to fully dissolve the polymer. After the solution was clear, a given 
amount of drug particles (1.5 g) was introduced into the solution. About $50 \%$ of the drug particles were fully dissolved into the acetone solution of the polymer; the rest remained suspended in the solution. Next $4 \mathrm{ml}$ of deionized water and $0.025 \mathrm{~g}$ of SDS as surfactant were added into the flask; note: GF is essentially insoluble in water. After 30 min of stirring, the flask containing the well-mixed solution/suspension was placed in a water bath at a constant temperature of $45^{\circ} \mathrm{C}$.

When the experiment was started, an aqueous solution of $50 \%$ by volume of ethylene glycol was circulated first through the shell side; a chiller (Polystat CR250WS, ColeParmer, Vernon Hills, IL, USA) cooled the glycol solution to $-9.1^{\circ} \mathrm{C}$ before introduction into the shell side. After about 2 min when the tube side wall was cooled down by the shell side cold liquid, the solution/suspension was fed into the lumen side of the SHFCC by a pump (Masterflex, model no. 7523-20, Cole-Parmer, Vernon Hills, IL, USA) at a rate of $3.5 \mathrm{ml} / \mathrm{min}$. The temperature indicated by thermocouple $\mathrm{T} 1$ was $45^{\circ} \mathrm{C}$; the feed side polymer solution containing the drug was cooled down in the lumen side of the module from $45^{\circ} \mathrm{C}$ to $5^{\circ} \mathrm{C}$ (unless otherwise mentioned) by the cold liquid on the shell side.

The polymer underwent precipitation from the solution and subsequently coated the pre-existing drug particles due to the rapid temperature reduction. Cooling also led to crystallization of some of the dissolved drug which was coated by the polymer as well. It is to be noted that the pre-existing suspended crystals acted also as seeds and may have experienced some growth. Coated drug crystals along with the excess polymer 
solution were swept out of the outlet of the SHFCC unit by the incoming feed solution since the SHFCC process was continuously operated. A vacuum microfiltration system (Omnipore Membrane, PTFE, $0.45 \mu \mathrm{m}$ pore size, $90 \mathrm{~mm}$ filter diameter; Millipore, Billerica, MA, USA) underneath the outlet was used to remove most of the solution exiting at a pressure of $\sim 1$ atm $(\sim 14.7 \mathrm{psia})$; the cake on the filter paper containing the coated particles was collected for post-treatment and characterization.

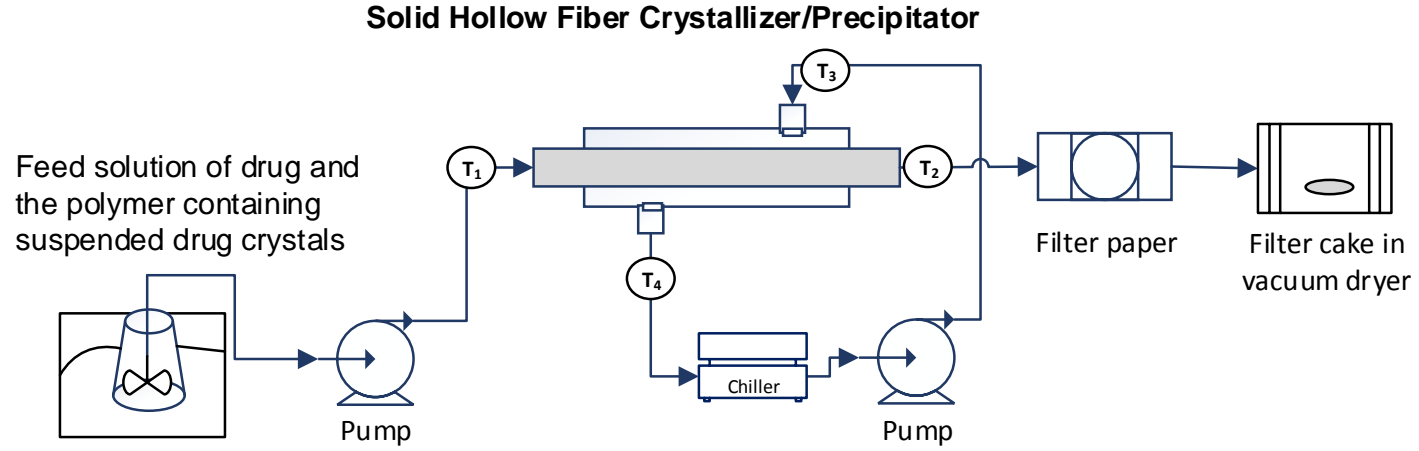

Fig. 2. Schematic diagram of solid hollow fiber crystallization setup for continuous polymer coating of drug particles.

Essentially no post-treatment step was needed except vacuum drying; the polymercoated crystals were free flowing.

\subsection{Characterization Methods}

The surface morphology of both coated and uncoated drug particles was analyzed by a Scanning Electron Microscope (SEM) (LEO 1530 Gemini, Zeiss, Thornwood, NY, USA). Dry sample powder was first subjected to carbon coating to make it conductive in order to prevent distortion of the micrographs by the charging of the sample.

To estimate the thickness of polymer coating on the drug particles, a Thermogravimetric Analyzer (TGA) (Pyris 1, PerkinElmer, Waltham, MA, USA) was employed to 
determine the mass loss of the drug component as well as the polymer coating component in any coated drug crystal. A dry sample was placed on a hang-down pan inside the furnace; the temperature of the sample was increased from $50^{\circ} \mathrm{C}$ to $550^{\circ} \mathrm{C}$ at a rate of $10^{\circ} \mathrm{C} / \mathrm{min}$; a $\mathrm{N}_{2}$ gas flow rate of $10 \mathrm{~cm}^{3} / \mathrm{min}$ was employed.

The X-ray diffraction (XRD) patterns of both coated and uncoated GF drug samples were measured in an X-ray Diffractometer by Empyrean (Philips, Westborough, MA, USA); the operating voltage and amperage were $45 \mathrm{kV}$ and $40 \mathrm{~mA}$, respectively. The sample powder was evenly dispersed on a sample holder; the surface was gently swept by a glass to maintain co-planarity in the area in order to get the accurate single source. The range of scanning was adjusted from $5^{\circ}$ to $45^{\circ} 2 \theta$ at a step size of $0.02^{\circ} 2 \theta$ and 15 ${ }^{\circ}$ time per step under the scanning of 255 detecting channels.

Using a Distek Dissolution tester (North Brunswick, NJ, USA), dissolution tests were run to compare the difference in dissolution rates between uncoated and coated GF drug particles. In these tests, $0.27 \%$ of SDS solution in $900 \mathrm{ml}$ volume was prepared as a buffer solution according to the USP II paddle method. The medium temperature was maintained at $37^{\circ} \mathrm{C}$. An amount equivalent to a dose of $50 \mathrm{mg}$ of $\mathrm{GF}$ was dropped into the dissolution vessel containing the buffer solution while a paddle stirring speed of 50 rpm was maintained. After 1, 3, 5, 10, 20, 30, 40, 50 and $80 \mathrm{~min}$, a syringe was used to extract $6 \mathrm{ml}$ volume of the solution and immediately analyzed by UV spectroscopy (52100 UV+, Cole-Parmer, Vernon Hills, IL, USA) at a wavelength of $296 \mathrm{~nm}$. The transmissivity of $0.27 \%$ SDS buffer solution was first analyzed as the benchmark 
before the test. The tests were repeated three times.

A Differential Scanning Calorimeter (DSC) (Model DSC 7, Perkin Elmer, Waltham, MA, USA) was applied to determine if the melting peaks in the DSC thermogram are the same before and after the polymer coating of drug particles. About $3 \mathrm{mg}$ of the sample was heated over a temperature range of $30-250^{\circ} \mathrm{C}$ at a rate of $10^{\circ} \mathrm{C} / \mathrm{min}$ with a $\mathrm{N}_{2}$ gas flow rate of $20 \mathrm{~cm}^{3} / \mathrm{min}$; the melting point was detected by Pyris software. The analysis was repeated three times using different batches.

The molecular structures of a drug crystal for both cases, uncoated/coated, were identified via a Raman Microscope (DXR, Thermo scientific, Waltham, MA, USA). For this measurement, the laser wavelength was set at $780 \mathrm{~nm}$; the laser power employed was $10 \mathrm{mw}$ for the run.

The particle size distribution was determined by a Laser Diffraction Particle Size Analyzer (LS230, Beckman Coulter, Brea, CA, USA). First an aqueous sample was prepared containing a suspension of the uncoated/coated drug particles. Next, this sample was placed under sonication for $30 \mathrm{~s}$ before the testing to break the soft bonding between coated particles.

\section{Results and Discussion}

\subsection{Crystallization of Griseofulvin Drug Particles in the SHFCC Module}

The first experiments checked out whether the drug GF can be crystallized in the SHFCC device when it is present in an acetone solution. Before such an experiment was conducted, a small amount of the dry GF powder as-received was put on a stub pin mount and characterized by SEM without any treatment. Figure 3(a) illustrates 
micrographs of the as-received pure GF particles at a particular magnification. The figure shows that the as-received drug particles have very rough surfaces and irregular shapes with a characteristic dimension of $\sim 10 \mu \mathrm{m}$.

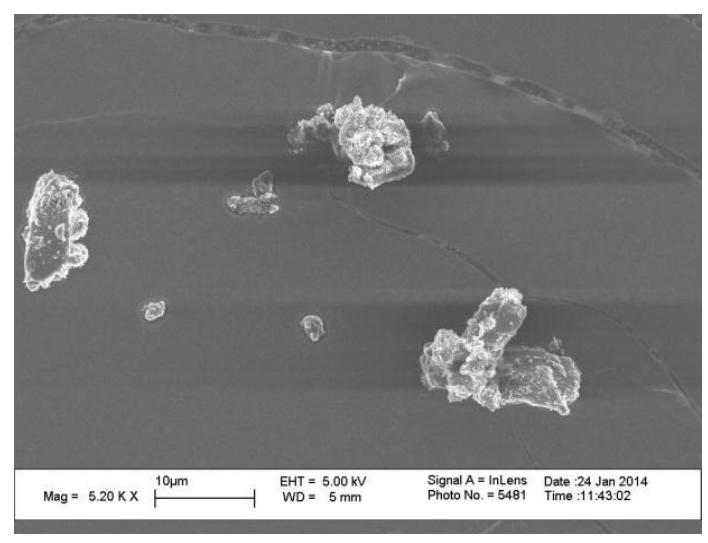

(a)

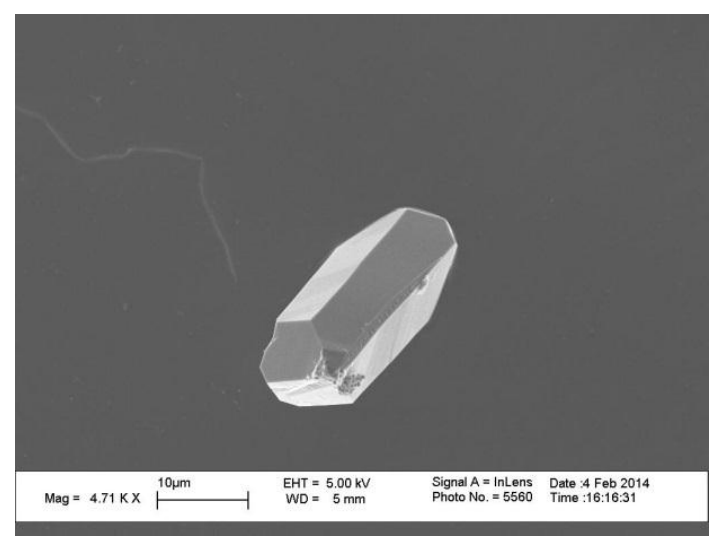

(b)

Fig. 3. SEM micrograph of (a) pure drug powder as-received and (b) GF crystal after precipitation in a SHFCC unit without any polymer.

To determine whether the SHFCC process will affect the characteristics of the drug particles produced by crystallization in the hollow fiber device, the as-received GF drug particles were dissolved in an acetone solution and subjected to crystallization by the SHFCC process without any polymer in solution. The GF particles were first fully dissolved in acetone in a flask ( $0.55 \mathrm{~g}$ GF in $20 \mathrm{ml}$ acetone, below the solubility limit) which was then submerged in a water bath at $45^{\circ} \mathrm{C}$ under stirring; after mixing for about 30 min when there were no drug particles suspended in the solution, the solution was passed through the tube side of the SHFCC device while the cold liquid was circulated in the shell side. Drug particles were collected at the outlet at the other end by a filter paper and subjected to various characterization steps after vacuum drying. 
Figure 3(b) shows a micrograph of a GF crystal obtained after pumping of the solution through the SHFCC device without the addition of Eudragit RL 100. Compared to Figure 3(a) where the drug shows no crystalline shape, the drug particle in Figure 3(b) appears to be a well-defined crystal after the SHFCC process. Although the surface shows some attached submicron size crystals, it is relatively smooth. After passing through the SHFCC acting as an efficient heat exchanger, GF crystals were formed from the solution due to rapid temperature reduction in the SHFCC module.

\subsection{Coating of Griseofulvin Drug Particles with Eudragit Polymer in the SHFCC Unit}

The acetone solution containing the dissolved polymer and the drug GF, along with the suspended drug particles, was pumped through the lumen of the hollow fine fibers in the SHFCC device. For a solution flow rate of $3.5 \mathrm{ml} / \mathrm{min}$ through the bore of 46 hollow fibers each having an internal radius of $0.021 \mathrm{~cm}$, the fluid velocity was $0.9 \mathrm{~cm} / \mathrm{s}$. The total length of each hollow fiber was $47 \mathrm{~cm}$ with about $40 \mathrm{~cm}$ being exposed to the cold shell-side liquid, the residence time was around $44 \mathrm{~s}$ (depends on the fiber length and the fluid velocity).

An evaluation of the crystalline products from the device outlet suggests that the drug crystallized first; the polymer precipitated later and covered the pre-existing drug crystals and the new drug crystals formed. Our earlier studies (Chen et al., 2014a; Chen et al., 2014b; Chen et al., 2015) have shown that Eudragit precipitation is a somewhat slow process regardless of the precipitation technique employed. No free uncoated crystals were visible. In Figure 4, a thin polymer coating film can be seen on each drug 
crystal. Compared to Figure 3(b) in which the drug particles were crystallized without any polymer being present in the solution, the surface morphology of the drug crystals appears to be different here which suggests that the polymer successfully encapsulated GF crystals after the SHFCC process.
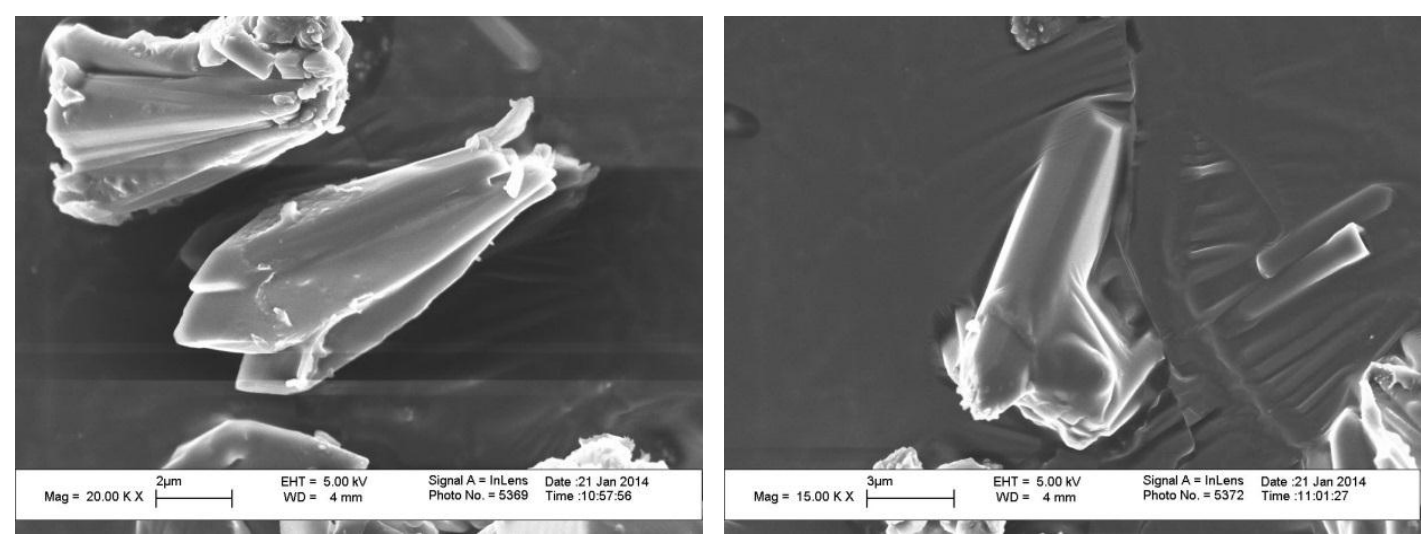

Fig. 4. SEM micrographs of polymer coated drug crystals after precipitation.

\subsection{TGA Characterization of Polymer Coated Drug Particles}

The thickness of the polymer coating on the drug crystals was determined by TGA. It involves measuring the \%weight loss of a sample as a function of increasing temperature. It can be used for polymer-coated drug particles to measure the weight of polymer coating. One has to identify the separate temperature ranges over which the weight loss was caused by drug decomposition and polymer decomposition since both the drug particles and the polymer will decompose leading to weight reduction shown in the TGA thermograms.

The polymer decomposition range can be identified from TGA graphs of prior experimental results of silica particles coated by the same polymer (Chen et al., 2014a; Chen et al., 2014b). For silica particles coated by Eudragit RL 100, since silica will not 
decompose during the heating up process, weight loss is almost completely contributed by polymer decomposition. Based on previous results (Chen et al., 2014a) for Eudragit RL 100, the primary temperature range of polymer decomposition is from 270 to $400^{\circ} \mathrm{C}$. Figure 5 shows the TGA results for the uncoated/coated drug particles obtained by the SHFCC technique in which the blue curve illustrates the results for the uncoated GF particles (as received) while the red dashed curve shows the TGA results for polymer coated GF particles obtained by the SHFCC technique. The decomposition range for the as-received GF is easily determined to be 150 to $270^{\circ} \mathrm{C}$. Based on this information, the actual polymer weight loss (mpolymer) can be estimated to be $13 \%$ (the y-axis value difference over the range of 270 to $400^{\circ} \mathrm{C}$ ) from the red dashed curve in Figure 5 so that the weight loss of GF particles ( $m_{\text {drug }}$ ) is therefore $87 \%$. This figure also includes the TGA results from the polymer-coated GF crystals (green dashed curve) obtained earlier by the PHFAC technique (Chen et al., 2015). It appears that the two TGA graphs are close with some minor differences especially at high temperatures.

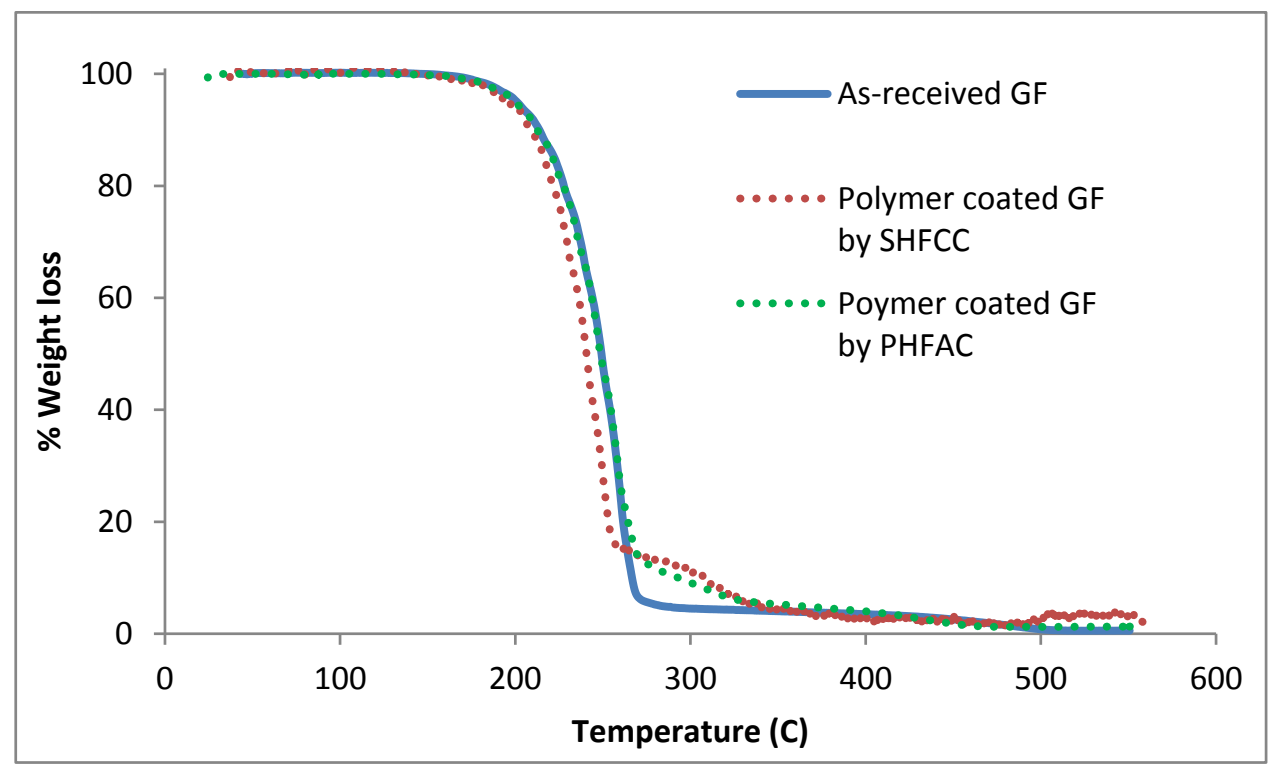


(b)

Fig. 5. TGA results of as-received GF, polymer-coated GF particles b obtained in the SHFCC and PHFAC devices.

From Figure 3, every drug crystal may be assumed before polymer coating to be a perfect rectangular parallelepiped having the three dimensions of $\mathrm{H}_{\mathrm{drug}}, \mathrm{W}_{\mathrm{drug}}$ and $\mathrm{L}_{\mathrm{drug}}$.

We assume also that a polymer coating of thickness $h$ covers the entire drug particle.

The polymer coating thickness on GF crystals may now be estimated from equation (1):

$$
\frac{m_{d r u g}}{m_{\text {polymer }}}=\frac{\rho_{\text {drug }} H_{d r u g} \times W_{d r u g} \times L_{d r u g}}{\rho_{\text {polymer }}\left[\left(H_{d r u g}+2 h\right) \times\left(W_{d r u g}+2 h\right) \times\left(L_{d r u g}+2 h\right)-H_{d r u g} \times W_{d r u g} \times L_{d r u g}\right]}
$$

Here the ratio $m_{d r u g} / m_{\text {polymer }}=(87 \% / 13 \%)=6.7$ was determined from the weight loss of the drug and the polymer from Figure 5. The densities of the drug particles and polymer are $\rho_{\text {drug }}(1.4 \mathrm{~g} / \mathrm{ml})$ and $\rho_{\text {polymer }}(1.1 \mathrm{~g} / \mathrm{ml})$, respectively. The height, width and length can be estimated from Figure 3(b) to be $17 \mu \mathrm{m}, 6 \mu \mathrm{m}$ and $8 \mu \mathrm{m}$, respectively. Based on these values, the polymer coating thickness is estimated from equation (1) to be $260 \mathrm{~nm}$. The corresponding thickness from the PHFAC process is around $75 \mathrm{~nm}$ (Chen et al., 2015).

The seemingly large estimate of the polymer coating thickness is likely to be due to a number of factors. First, Figure 4 shows that there is a significant amount of agglomeration of the coated crystals. The polymers used to bridge the neighboring crystals will contribute to a higher estimate of the coating thickness around any crystal. Secondly, the estimates of crystal dimensions obtained from Figure 3(b) are larger than those in Chen et al. (2015). Correspondingly from equation (1) the coating thickness 
will be higher. We should also add that the preexisting crystals in suspension will grow since they will act as seeds before they get coated by the polymer.

In our earlier publications (Chen et al., 2014a; Chen et al., 2015), the coating polymer Eudragit RL 100 used has the following elements: C, O, H, N and $\mathrm{Cl}$; the core particles in those studies were of silica which contains only the elements of Si and O. Therefore the techniques of STEM and EDS can easily determine the possible coating thickness since the elements making up the coating polymer and core particles are mostly different. Here, however, the core drug particle of Griseofulvin contains the elements of $\mathrm{C}, \mathrm{O}, \mathrm{H}$ and $\mathrm{Cl}$ which are also identical to the elements in the coating polymer. The only difference is that the coating polymer contains the extra element $\mathrm{N}$ but the amount of $\mathrm{N}$ is too little to be detected efficiently. That is why STEM and EDS-based measurements were not carried out here to estimate the coating thickness. It is to be recognized that there are additional methods of estimating the coating thickness such as Near-infrared Spectroscopy (NIR) (Lee et al., 2011).

\subsection{XRD and Raman Spectroscopy}

To identify the crystal structure of the sample, XRD analysis for coated and uncoated GF particles was performed. The results are shown in Figure 6. There was no alteration in any peak position indicating that the polymer coating of the drug particles did not affect the crystal structure of GF since the characteristic peaks of the two samples were identical. A slight change in the peak intensity in the coated sample is due to the thick polymer coating around the coated particles which could weaken the signals. The 
Raman spectra for the uncoated/coated drug crystals at the shift range from 0 to 3500 $\mathrm{cm}^{-1}$ were also determined. In line with the XRD results, the characteristic peaks for both uncoated GF and coated GF particles are all the same. This suggests that coating of the drug particles by precipitation of the polymer from the solution by the SHFCC process did not alter the drug molecular structure; therefore the drug should retain its pharmaceutical properties even after coating with the polymer.

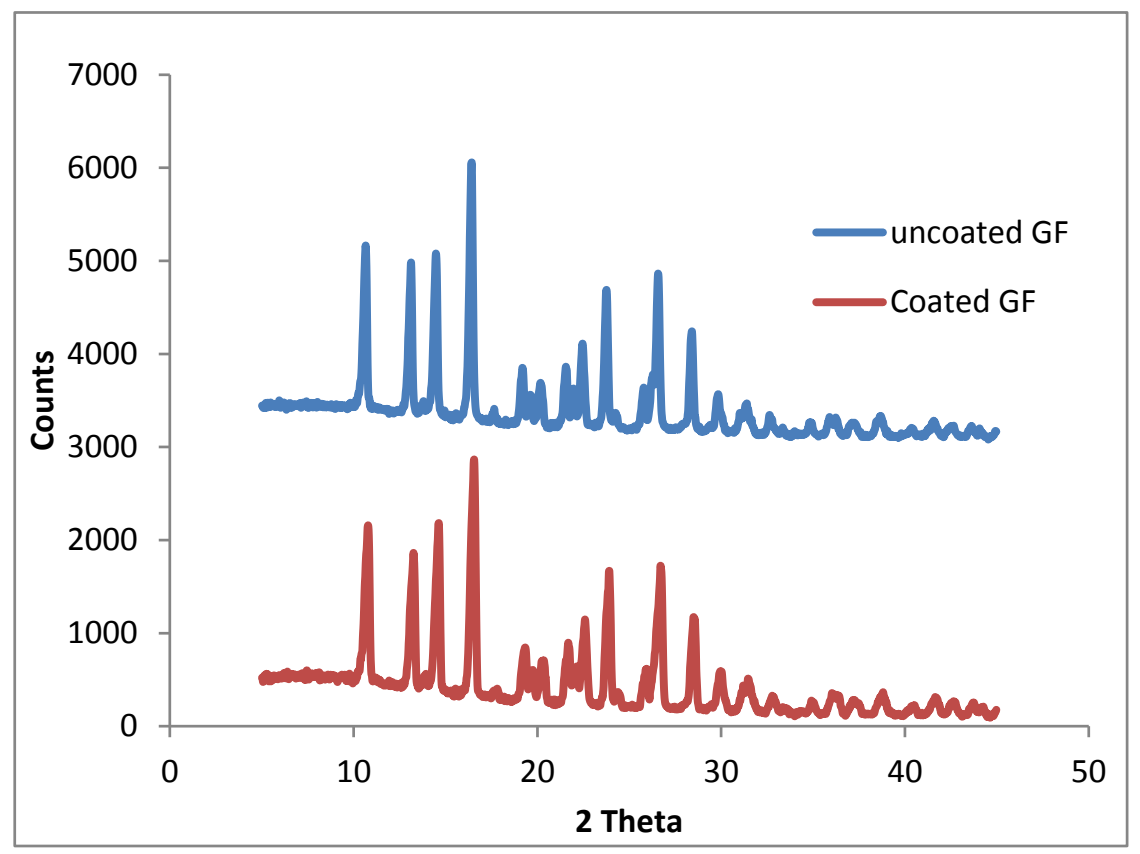

Fig. 6. X-ray diffractograms of uncoated GF and polymer-coated GF samples.

\subsection{Differential Scanning Calorimetry (DSC)}

Differential scanning calorimetry analysis was also performed for the as-received GF and the Eudragit coated GF crystals; the results are shown in Figure 7. The peak position of $220.20^{\circ} \mathrm{C}$ determined by the Pyris software for the as-received Griseofulvin particles was almost identical to the peak value of $221.37^{\circ} \mathrm{C}$ for the polymer coated GF. The slight intensity difference observed may be due to the polymer coating covering 
the entire drug particles that prevents the signal from penetration. The melting peak corresponds to the maximum peak as shown in the figure.

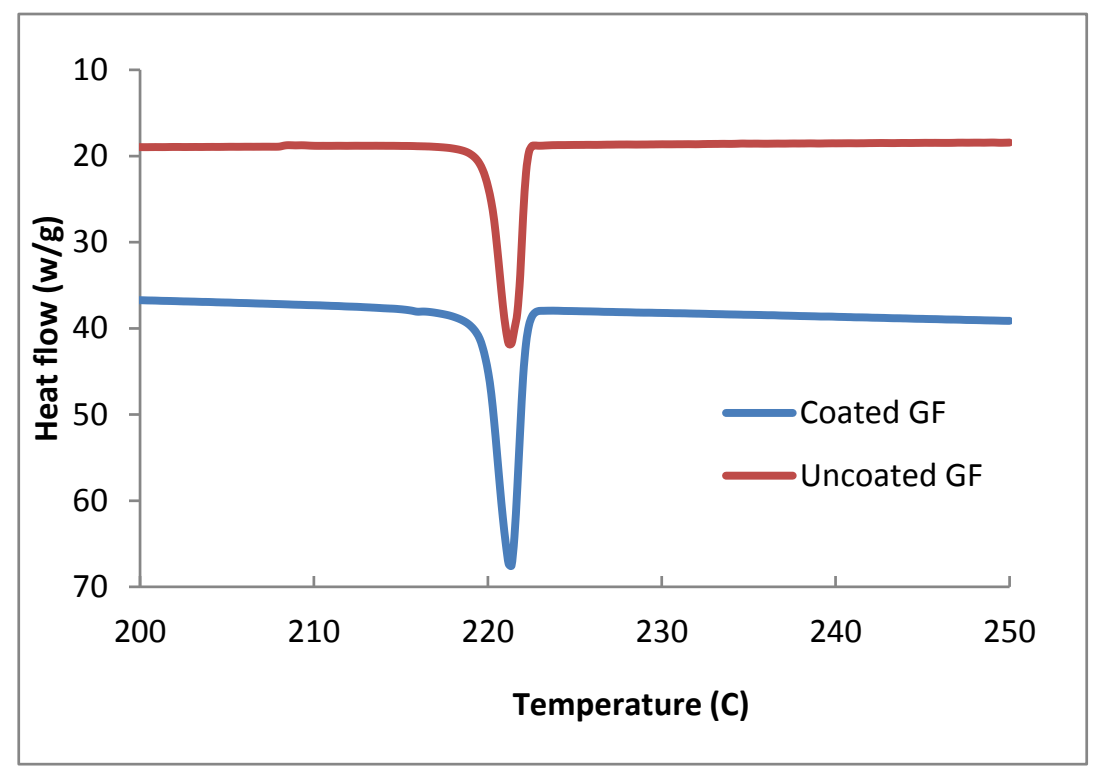

Fig. 7. Differential scanning calorimetry patterns for uncoated GF and coated GF.

\subsection{Dissolution Testing Study}

To identify the effect on controlled release of the drug GF when it was coated by the polymer using solid hollow fiber membrane-based cooling crystallization method, dissolution studies of the as-received drug, uncoated drug and the polymer coated drug were carried out. The dissolution test results are shown in Figure 8.

The dissolution profiles for the as-received GF and the uncoated GF crystals are very similar; these results suggest that even when GF was recrystallized from the solution by the SHFCC device, it did not change the estimate of the time for the drug to get $100 \%$ dissolved in the buffer solution. The dissolution profile of the coated GF crystals (green curve) is however completely different from the two curves of the uncoated crystals. Only about $18 \%$ of the coated GF crystals dissolved even after 80 min which implied 
that the polymer coating was acting as a barrier to prevent the drug component from getting quickly dissolved in the solution. The slight difference between the as-received GF and uncoated GF crystals between 5 and 30 minutes may have resulted from different structures of the uncoated GF and the as-received GF. The overall size of the uncoated GF and the as-received GF are comparable.

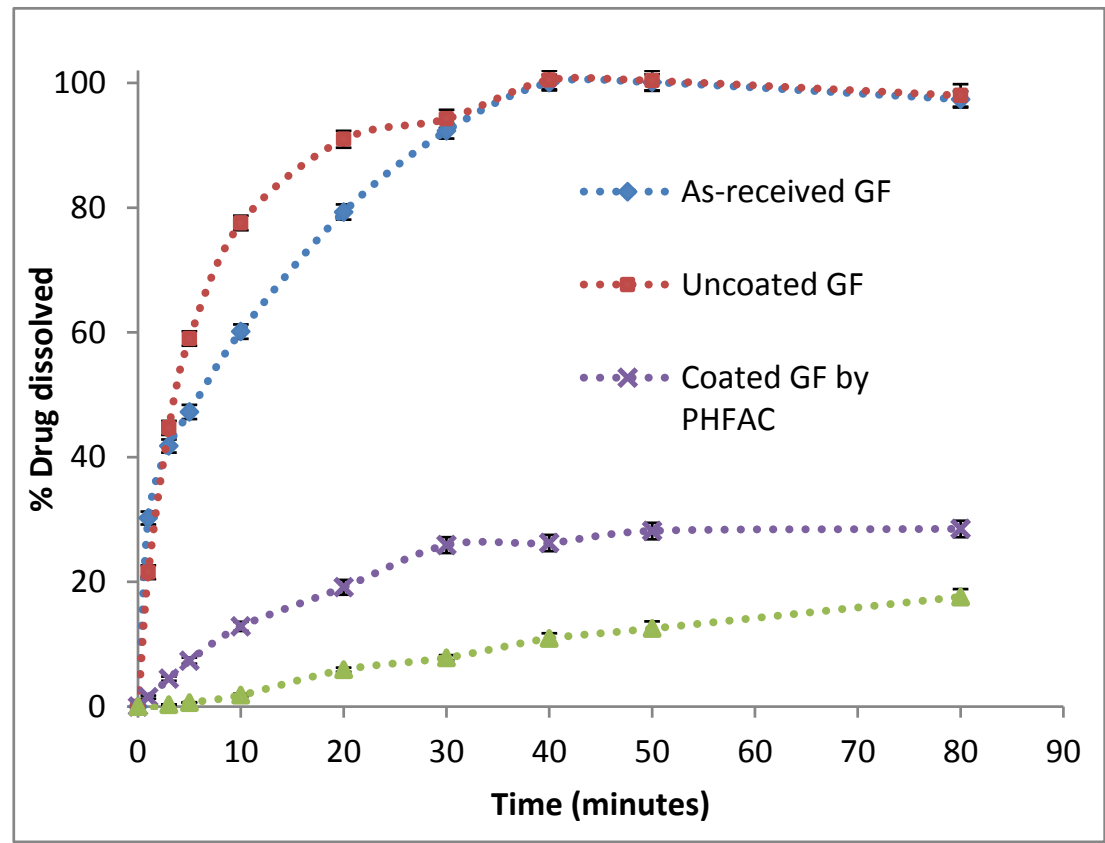

Fig. 8. Dissolution profiles for crystals of as-received GF, uncoated GF, and polymer coated GF (green data points); the blue data points are from Chen et al. (2015) using the PHFAC technique.

We have also incorporated in Figure 8 the dissolution data for polymer-coated crystals of GF obtained by the PHFAC technique (blue curve) (Chen et al., 2015). The rates of dissolution of these samples were faster in that $20 \%$ of the drug was dissolved by 50 minutes. The differences between the two results are due to the thicker coating in this study $(260 \mathrm{~nm})$ as well as larger size of the coated crystals of this study. In Chen et al. (2015), the length, width and height of representative drug crystal samples were 10, 2 , and $2 \mu \mathrm{m}$ respectively. These values are significantly smaller than those of the samples 
of the present study; correspondingly the dissolution times in the present study are longer.

\subsection{Crystal Size Distribution Measurements}

Using the LS 230 laser diffraction analyzer, the crystal size distributions (CSD) of the as-received uncoated drug particles as well as Eudragit RL 100 coated Griseofulvin drug particles collected from the SHFCC process were measured; the objective was to compare the level of agglomeration before and after coating. Figure 9 illustrates the CSD profiles of the uncoated and coated drug crystals in which the median size of the as-received GF $(11.6 \mu \mathrm{m})$ was about 3 times smaller than that of the polymer encapsulated GF $(31.19 \mu \mathrm{m})$. There are two possible reasons for this behavior. The much larger median size of the polymer-coated drug crystals is very likely due to the agglomeration between coated particles since the precipitated polymer will form bridges attaching neighboring drug particles together. That the preexisting drug particles in suspension will grow since they are acting also as seeds for the crystallizing solution of GF is another factor contributing to the larger size of the polymer-coated crystals. 


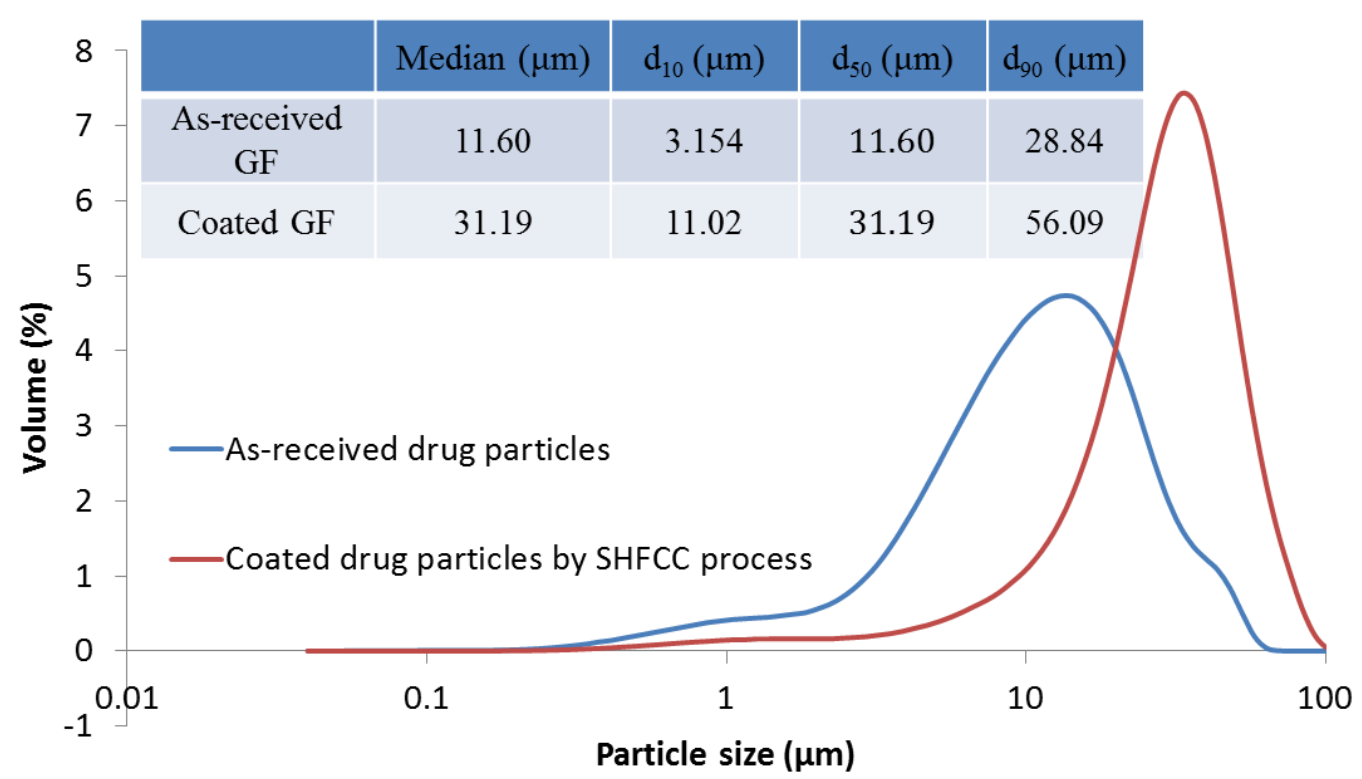

Fig. 9. Crystal size distribution of as-received and polymer-coated Griseofulvin drug particles.

\subsection{Comparison with the PHFAC Process}

The polymer Eudragit RL 100 was used earlier to develop a continuous process of synthesizing polymer-coated GF crystals from a solution of the polymer and the drug in acetone by using the anti-solvent water in a porous hollow fiber membrane-based device (Chen et al., 2015). It is useful to compare the nature of this product with that obtained in this work. First, the crystals appear to be quite similar. Second, the XRD and Raman spectra appear to be identical in both cases. Third, in both cases, the rate of dissolution of the drug was substantially reduced by the polymer coating; here the reduction is larger because the coating is thicker, $260 \mathrm{~nm}$ compared to $75 \mathrm{~nm}$ in (Chen et al., 2015); as pointed out earlier larger crystal sizes in this study also makes a difference. A major difference between the two techniques lies in the amount of polymer precipitated and the drug crystallized out of the solution. In the PHFAC technique, the level of supersaturation that is developed and the rate at which it is 
developed is very high unless appropriate effort is made to counter it. Therefore the extent of utilization of the dissolved drug and the polymer is very high. In the present SHFCC technique, it is significantly slower leading to a lower rate of utilization of the dissolved material. One can choose to apply either technique to coat the drug particles depending on different operating conditions and requirements. They are both economical, practical, easy to scale-up and do not require demanding operating conditions compared to conventional coating techniques.

\section{Conclusions}

A novel approach for continuous production of polymer coated drug particles has been developed by using the solid hollow fiber cooling crystallization (SHFCC) technique. Pre-existing drug particles of Griseofulvin (GF) in suspension as well as GF crystals which crystallized from the GF solution-suspension in acetone were coated by the polymer Eudragit RL 100. The polymer precipitated from the acetone solution as it was cooled from $45^{\circ} \mathrm{C}$ to around $5^{\circ} \mathrm{C}$ while flowing in the lumen of the solid hollow fine fibers in the SHFCC module. Tests using XRD, Raman and DSC indicated that the polymer coating covering the drug particles did not influence the pharmaceutical signatures of the drug. The dissolution test results suggest that the coated drug demonstrated a controlled release potential since the polymer-coated drug showed a much slower dissolution rate with time compared to the uncoated drug.

This novel method for polymer coating of drug particles should be attractive for the pharmaceutical industry in the area of drug delivery. The relatively simple set up and 
the modest requirements for experimental conditions provide an economic advantage compared to other coating techniques. The ability to run continuously in contrast to presently available coating techniques which are run in a batch mode also suggests a possibility of mass production.

\section{Acknowledgements}

The authors gratefully acknowledge support for this research from the National Science Foundation through Grant CMMI-1100622. We thank the Bristol-Myers Squibb Corporation (BMS), and especially Dr. San Kiang of BMS, for providing us with an industrial perspective.

\section{References}

Bernkop-Schnürch, A., Scholler, S., Biebel, R. G., 2000. Development of Controlled Drug Release Systems Based on Thiolated Polymers. Journal of Controlled Release. 66 (1), 39-48.

Bhattacharyya, S., Wang, H., Ducheyne, P., 2012. Polymer-coated Mesoporous Silica Nanoparticles for the Controlled Release of Macromolecules. Acta Biomaterialia. 8 (9), 3429-3435.

Chen, D.C., Singh, D., Sirkar, K.K., Pfeffer, R., 2014a. Continuous Polymer Coating/encapsulation of Submicron Particles using a Solid Hollow Fiber Cooling Crystallization Method. I\&E Chem. Res. 53, 6388-6400.

Chen, D.C., Singh, D., Sirkar, K.K., Zhu, J., Pfeffer, R., 2014b. Continuous Polymer Nanocoating on Silica Nanoparticles. Langmuir. 30, 7804-7810.

Chen, D.C., Singh, D., Sirkar, K.K., Pfeffer, R., 2015. Continuous Synthesis of Polymer-Coated Drug Particles by Porous Hollow Fiber Membrane-Based Antisolvent Crystallization, Langmuir. 31(1), 432-441.

Cui, L., Wang, R., Ji, X., Hu, M., Wang, B., Liu, J., 2014. Template-assisted Synthesis of Biodegradable and $\mathrm{pH}$-responsive Polymer Capsules via RAFT Polymerization for Controlled Drug Release. Materials Chemistry and Physics. 148 (1), 87-95.

Falk, R., Randolph, T.W., Meyer, J.D., Kelly, R.M., Manning, M.C., 1997. Controlled Release of Ionic Compounds from Poly (L-lactide) Microspheres Produced by Precipitation with a Compressed Antisolvent. J. Control. Release. 44, 77-85. 
Fluri, D. A., Kemmer, C., Daoud-El Baba, M., Fussenegger, M., 2008. A Novel System for Trigger-controlled Drug Release from Polymer Capsules. Journal of Controlled Release. 131 (3), 211-219.

Ghebaur, A., Garea, S. A., Iovu, H., 2012. New Polymer-halloysite Hybrid Materialspotential Controlled Drug Release System. International Journal of Pharmaceutics. 436 (1), 568-573.

Kakizawa, Y., Nishio, R., Hirano, T., Koshi, Y., Nukiwa, M., Koiwa, M., Michizoe, J., Ida, N., 2010. Controlled Release of Protein Drugs from Newly Developed Amphiphilic Polymer-based Microparticles Composed of Nanoparticles. Journal of Controlled Release. 142 (1), 8-13.

Kim, J.H., Paxton, T.E., Tomasko, D.L., 1996. Microencapsulation of Naproxen using Rapid Expansion of Supercritical Solutions. Biotechnol. Prog. 12, 650-661.

Laga, R., Carlisle, R., Tangney, M., Ulbrich, K., Seymour, L. W., 2012. Polymer Coatings for Delivery of Nucleic Acid Therapeutics. Journal of Controlled Release. 161 (2), 537-553.

Lai, S. K., Wang, Y.Y., Hanes, J., 2008. Mucus-penetrating Nanoparticles for Drug and Gene Delivery to Mucosal Tissues. Adv. Drug Deliv. Rev. 61 (2), 158-171.

Langer, R., 1990. New Methods of Drug Delivery, Science, 249, 1527-1533.

Lee M. J., Seo, D.Y., Lee, H.E., Wang, I.C., Kim, W.S., Jeong, M.Y., Choi, G.J., 2011, In-line NIR Quantification of Film Thickness on Pharmaceutical Pellets during a Fluid Bed Coating Process. International Journal of Pharmaceutics. 403(1-2), 66-72.

Li, L., Wang, L., Li, J., Jiang, S., Wang, Y., Zhang, X., Ding, J., Yu, T., Mao, S., 2014. Insights Into the Mechanisms of Chitosan-anionic Polymers-based Matrix Tablets for Extended Drug Release. International Journal of Pharmaceutics. 476 (1), 253-265.

Mei, X., Chen, D., Li, N., Xu, Q., Ge, J., Li, H., Lu, J. 2012. Hollow Mesoporous Silica Nanoparticles Conjugated with $\mathrm{pH}$-sensitive Amphiphilic Diblock Polymer for Controlled Drug Release. Microporous and Mesoporous Materials. 152, 16-24.

Midler, M., Paul, E. L., Whittington, E. F., Futran, M., Liu, P. D., Hsu, J., Pan, S.H., 1994. Crystallization Method to Improve Crystal Structure and Size. US Patent 5, $314,506$.

Montes, A., Gordillo, M.D., Pereyra, C., Martinez de la Ossa, E.J., 2012. Polymer and Ampicillin Co-precipitation by Supercritical Antisolvent Process. J. Supercritical Fluids. 63, 92-98.

Myerson, A.S., 2002. Handbook of Industrial Crystallization. 2nd Ed. ButterworthHeinemann, Boston.

Nahrup, J. S., Gao, Z. M., Mark, J. E., Sakr, A., 2004. Poly (dimethylsiloxane) Coatings for Controlled Drug Release-polymer Modifications. International Journal of Pharmaceutics. 270 (1), 199-208.

Nance, Elizabeth A., Woodworth, Graeme F., Sailor, Kurt A., Shih, Ting-Yu., Xu, Qingguo., Swaminathan, Ganesh., Xiang, Dennis., Eberhart, Charles., Hanes, 
Justin., 2012. A Dense Poly (Ethylene Glycol) Coating Improves Penetration of Large Polymeric Nanoparticles Within Brain Tissue. Science Translational Medicine. 4 (149), 149ra119.

Paulsson, M., Edsman, K., 2002. Controlled Drug Release from Gels Using

Lipophilic Interactions of Charged Substances with Surfactants and Polymers. Journal of Colloid and Interface Science. 248 (1), 194-200.

Ramyadevi, D., Rajan, K. S., 2015. Interaction and Release Kinetics Study of Hybrid Polymer Blend Nanoparticles for $\mathrm{pH}$ Independent Controlled Release of an Antiviral Drug. Journal of the Taiwan Institute of Chemical Engineers. 50, 1-11.

Song, L.M., Li, B., Zarkadas, D., Christian, S., Sirkar, K. 2010. Polymeric Hollow Fiber Heat Exchangers for Thermal Desalination Processes. I\&E Chem. Res., 49, 11961-11977.

Sosa, M.V., Rodriguez-Rojo, S., Mattea, F., Cismondi, M., Cocero, M.J., 2011. Green Tea Encapsulation by Means of High Pressure Antisolvent Coprecipitation. J. Supercritical Fluids. 56, 304-311

Sundaresan, V., Menon, J. U., Rahimi, M., Nguyen, K. T., Wadajkar, A. S., 2014. Dual-responsive Polymer-coated Iron Oxide Nanoparticles for Drug Delivery and Imaging Applications. International Journal of Pharmaceutics. 466 (1), 1-7.

Tavare, N.S., 1989. Micromixing Limits in an MSMPR Crystallizer. Chemical Engineering Technology. 12, 1-12.

Tsutsumi, A., Nakamoto, S., Mineo, T., Yoshida, K., 1995. A Novel Fuidized-bed Coating of Fine Particles by Rapid Expansion of Supercritical Fluid Solutions. Powder Technol. 85, 275-278.

Wang, Y., Dave, R.N., Pfeffer, R., 2004. Polymer Coating/encapsulation of Nanoparticles using a Supercritical Anti-solvent Process. J. Supercritical Fluids. $28,84$.

Wulff, R., Leopold, C. S., 2014. Coatings from Blends of Eudragit ${ }^{\circledR}$ RL and L55: A Novel Approach in $\mathrm{pH}$-controlled Drug Release. International Journal of Pharmaceutics. 476 (1), 78-87.

Zahran, F., Cabanas, A., Cheda, J., Renuncio, J., Pando, C., 2014. Dissolution Rate Enhancement of the Anti-inflammatory Drug Diflunisal by Coprecipitation with a Biocompatible Polymer Using Carbon Dioxide as a Supercritical Fluid Antisolvent. J. Supercritical Fluids. 88, 56-65.

Zarkadas, D.M., Sirkar, K.K., 2004. Solid Hollow Fiber Cooling Crystallization. Ind. Eng. Chem. Res. 43, 7163-7180.

Zarkadas, D.M., Sirkar, K. K., 2006. Antisolvent Crystallization in Porous Hollow Fiber Devices. Chem. Eng. Sci. 61, 5030- 5048. 
Solid Polymeric Hollow

Fiber Wall

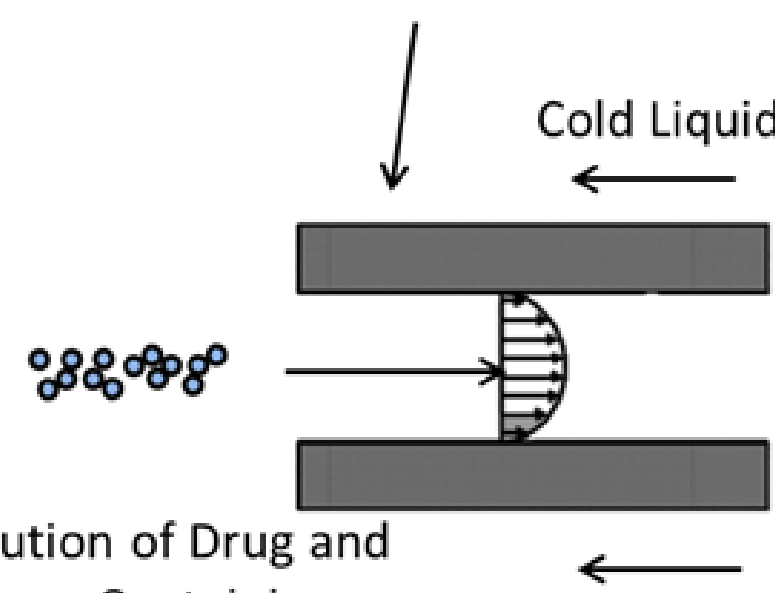

Feed Solution of Drug and the Polymer Containing Suspended Drug Crystals

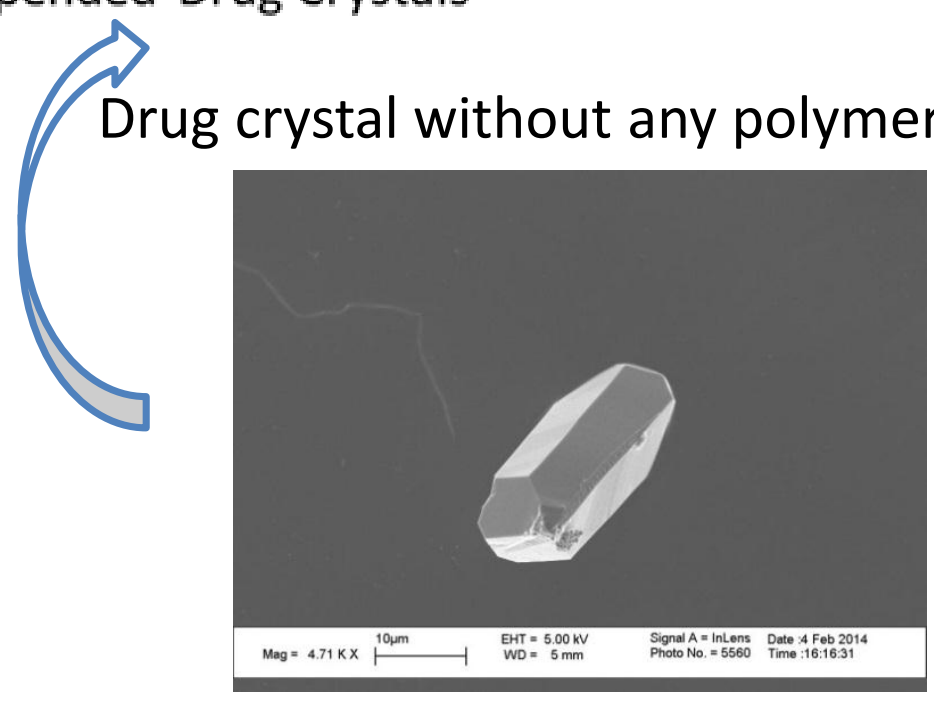

Solid Hollow Fiber Cooling

Crystallizer

Cold Liquid in $\mathrm{T}=-9.1^{\circ} \mathrm{C} \downarrow$
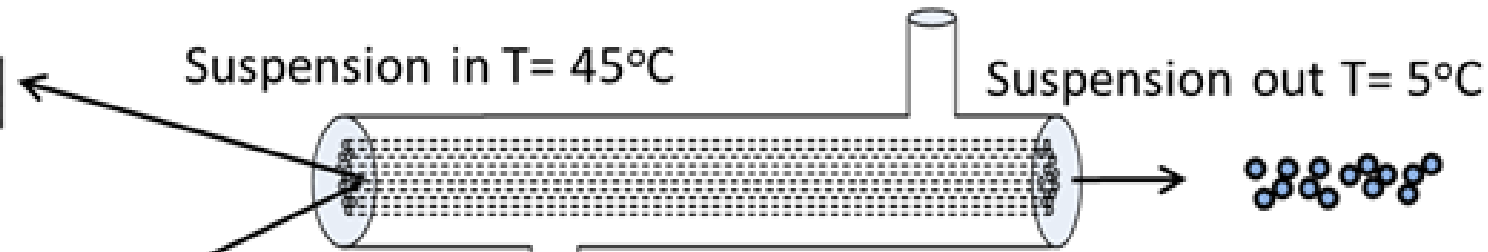

Cold Liquid out $\mathrm{T}=-9^{\circ} \mathrm{C}$
Suspension of Polymercoated Drug Crystals Out

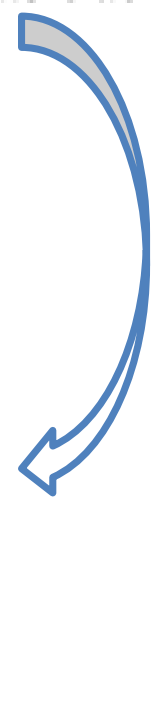

Polymer coating of drug crystals in a solid hollow fiber cooling crystallizer (SHFCC) 